\title{
Possible roles of COX-1 in learning and memory impairment induced by traumatic brain injury in mice
}

\author{
J.L. Shang ${ }^{1}$, Q. Cheng ${ }^{2}$, W.F. Yang ${ }^{3}$, M. Zhang ${ }^{1}$, Y. Cui ${ }^{1}$ and Y.F. Wang ${ }^{1}$ \\ ${ }^{1}$ Neurology Department, Peace Hospital Attached to Changzhi Medical College, Changzhi, China \\ ${ }^{2}$ Neurology Department, Changzhi City People's Hospital, Changzhi, China \\ ${ }^{3}$ Neurology Department, People's Hospital of Dali, Dali, China
}

\begin{abstract}
People who suffer from traumatic brain injury (TBI) often experience cognitive deficits in spatial reference and working memory. The possible roles of cyclooxygenase-1 (COX-1) in learning and memory impairment in mice with TBI are far from well known. Adult mice subjected to TBI were treated with the COX-1 selective inhibitor SC560. Performance in the open field and on the beam walk was then used to assess motor and behavioral function 1, 3, 7, 14, and 21 days following injury. Acquisition of spatial learning and memory retention was assessed using the Morris water maze on day 15 post-TBI. The expressions of COX-1, prostaglandin E2 (PGE2), interleukin (IL)-6, brain-derived neurotrophic factor (BDNF), platelet-derived growth factor BB (PDGF-BB), synapsin-I, and synaptophysin were detected in TBI mice. Administration of SC560 improved performance of beam walk tasks as well as spatial learning and memory after TBI. SC560 also reduced expressions of inflammatory markers IL-6 and PGE2, and reversed the expressions of COX-1, BDNF, PDGF-BB, synapsin-I, and synaptophysin in TBI mice. The present findings demonstrated that COX-1 might play an important role in cognitive deficits after TBI and that selective COX-1 inhibition should be further investigated as a potential therapeutic approach for TBI.
\end{abstract}

Key words: Traumatic brain injury; COX-1; Learning and memory; SC560

\section{Introduction}

Traumatic brain injury (TBI), a serious health problem, is among the leading causes of acute and chronic disability in China and around the world. TBI can cause intellectual and cognitive deficits and mood and behavioral changes both short and long term $(1,2)$. Despite the intense effort and billions of dollars invested for therapeutic measures, treatment of $\mathrm{TBI}$ is limited and far from satisfactory.

Cyclooxygenase (COX)-1, one of the major isoforms of $\mathrm{COX}$, is the key rate-limiting enzyme in the synthesis of prostaglandin (PG) from arachidonic acid and is thought to maintain essential physiological functions $(3,4)$. Although there are a limited number of studies examining the function of COX-1 expressed in the brain, it is clear that the pattern of COX-1 expression in the central nervous system (CNS) is distinct from the constitutive expression observed in peripheral tissues. It has been reported that COX-1 expression within and adjacent to the developing injury was significantly increased in rodents and humans $(5,6)$. Moreover, the significant increase in COX-1 protein expression in these patients with $\mathrm{TBI}$ was observed in vessel endothelial-smooth muscle cells, and CD68+ microglia/macrophages strictly confined to the lesion (6). However, the possible roles of COX-1 involved in learning and memory impairment of mice that suffered from TBI is still unknown.

The aim of the present study was to test the hypothesis that COX-1 is involved in learning and memory deficits induced by TBI in mice. Adult mice were subjected to TBI induced by a weight-drop model and were treated with the COX-1 selective inhibitor 5-(4-chlorophenyl)-1-(4-methoxyphenyl)-3-trifluoromethylpyrazole (SC560). Afterward, open field and beam walk performance were used to assess motor and behavioral function 1, 3, 7, 14, and 21 days postoperation (dpo). Acquisition of spatial learning and memory retention was assessed using the Morris water maze test on day 15 post-TBI. The expressions of COX-1 and the learning-associated neurotrophic factors (NTFs), brain-derived neurotrophic factor (BDNF) and

Correspondence: Y.F. Wang, Neurology Department, Peace Hospital Attached to Changzhi Medical College, Changzhi 046000, China. Fax: +86-355-302-1477. E-mail: ssf0221@163.com 
platelet-derived growth factor BB (PDGF-BB), as well as the synaptic proteins synapsin-I and synaptophysin (SYP) were detected by Western blot analysis in the hippocampus of TBI mice. Moreover, expression of interleukin-6 (IL6) and PGE2 levels in peripheral blood (PB) of mice was also measured by enzyme-linked immunosorbent assay (ELISA).

\section{Material and Methods}

\section{Animal grouping}

This investigation conformed to the Guide for the Care and Use of Laboratory Animals published by the United States National Institutes of Health (Bethesda, MD, USA; NIH Publication No. 85-23, revised 1996).

C57BL/6J mice (16 weeks old) were randomly divided into 4 groups ( $n=8 /$ group) as follows: sham group, mice underwent sham surgery but without actual induction of TBI; TBI group, mice were injured using the TBI device; vehicle group, TBI mice were treated with $0.2 \mathrm{~mL} 20 \%$ dimethyl sulfoxide (DMSO; Roche, USA) by intraperitoneal injection; SC560-treated group, TBI mice received intraperitoneal injection of SC560 (5 mg/kg body weight; Cayman Chemical, USA) every day post-TBI surgery. SC560 (R\&D Systems, USA) was dissolved in DMSO and diluted with saline to a final concentration of $20 \%(\mathrm{v} / \mathrm{v})$ DMSO.

\section{TBI model}

Animals underwent anesthesia with $3.6 \%$ chloral hydrate by intraperitoneal injection and fixed on a stereotactic platform. The skull was then opened and the corticomotor area in the cerebral cortex was exposed. A mouse model of TBI was developed using a device that produces controlled cortical impact $(\mathrm{CCl})$, permitting independent manipulation of tissue deformation and impact velocity. The left parietotemporal cortex was subjected to moderate $\mathrm{CCl}(2 \mathrm{~mm}$ tissue deformation and $6.0 \mathrm{~m} / \mathrm{s}$ ) or sham surgery (7). After injury, the incision was closed with interrupted 6-0 silk sutures, anesthesia was terminated, and the animal was placed into a heated cage to maintain normal core temperature for 45 min postinjury. All animals were monitored carefully for at least $4 \mathrm{~h}$ after surgery and then daily. Surgeries for individual studies were performed by the same model expert within a short time frame (3 days) to minimize experimental variation, with the control and treated groups randomly intermingled.

\section{Functional evaluation of behavior}

Open field test. Gross motor ability was measured using a novel open field paradigm. Briefly, mice were placed in a novel, brightly lit, circular arena. A TOPSCAN tracking system (Clever Sys Inc., USA) was used to quantify ambulation distance over a 5-min trial period.

Beam walk. Fine motor coordination was evaluated for all animals using a beam walk task as described before (8), which was performed at 1, 3, 7, 14, and $21 \mathrm{dpo}$.
Morris water maze. A Morris water maze paradigm was employed to assess spatial learning by training mice to locate a hidden, submerged platform using examination of visual information and was conducted exactly as described previously (8). The test was conducted 15-20 days after injury, and each mouse was tested for 3 trials per day for 6 consecutive days. The time required (escape latency) to find the hidden platform with a 90-s limit was recorded by a blinded observer and tracked using TOPSCAN (Clever Sys Inc.). A probe trial of $90 \mathrm{~s}$ was given 1 day after the final learning trial. The percentage of time spent in the quadrant where the platform was previously located was recorded. The number of entries into the correct quadrant was also recorded.

\section{Sample preparation}

At $1,3,7,14$, and $21 \mathrm{dpo}$, a total of $250 \mu \mathrm{L}$ PB was obtained from the tails of the mice in each group before the mice were killed. The nucleate cells were isolated from PB and then processed for analysis of IL-6 levels by ELISA assay. Then, the hippocampus of the injured brain was extracted from mice in each group, and these tissues were prepared for Western blot analysis.

\section{Western blot analysis}

Western blot analysis was performed as previously described (9). Briefly, after carefully rinsing in cooled phosphate-buffered saline, the tissues were homogenized on ice in a lysis buffer (Pierce Biotechnology, Thermo Scientific, USA) and centrifuged at 10,000 $\mathrm{g}$ for $30 \mathrm{~min}$. The absorbance of the protein bands for COX-1 (1:1000; Santa Cruz, USA), BDNF (1:200; Santa Cruz), PDGF-BB (1:800; Santa Cruz), synapsin-I (1:400; Santa Cruz), and SYP (1:400; Santa Cruz) were analyzed for each group in order to semiquantitatively assess these protein levels. GAPDH (1:800; Santa Cruz) was used as internal control.

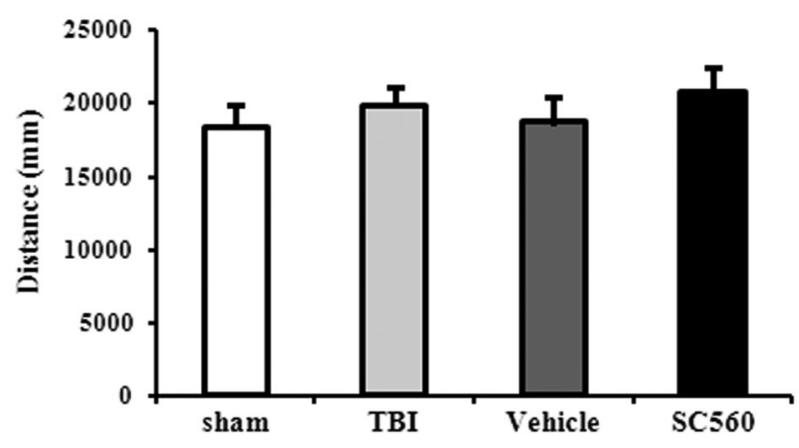

Figure 1. Effects of SC560 treatment in the traumatic brain injury (TBI) mouse model evaluated by the open field. Data are reported as means $\pm S D(n=8)$. dpo: days post operation. Neither TBI nor SC560 treatment altered the gross motor ability detected by the open-field test. 


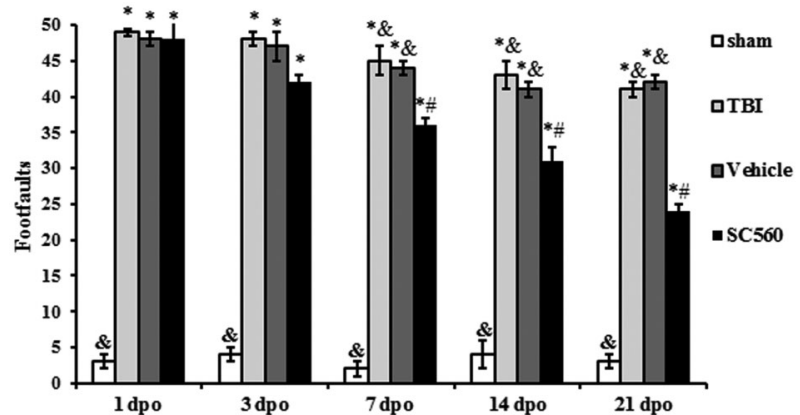

Figure 2. Effects of SC560 on fine motor coordination evaluated by the beam walk test after traumatic brain injury (TBI). TBI caused significant deficits in fine motor coordination (sham vs TBI and vehicle groups, $\mathrm{P}<0.001$ ). However, there was no significant difference between the TBI group and the vehicle group throughout the experiment $(P>0.05)$. SC560 treatment significantly altered beam walk performance from $7 \mathrm{dpo}$ compared with that of the TBI and vehicle groups $(P<0.05)$. Data are reported as means $\pm S D$ $(n=8)$. dpo; days post operation. ${ }^{\&} P<0.05$, compared to SC560treated group; ${ }^{*} \mathrm{P}<0.05$, compared to sham group; ${ }^{\#} \mathrm{P}<0.05$, compared to vehicle-treated group (repeated-measures ANOVA with post hoc analyses performed after any significant main effect).

\section{ELISA}

Expression of IL-6 protein was detected according to the manufacturer's protocol by using a mouse IL-6 sandwich ELISA kit (Millipore, Cat. No. EZMIL6, Australia). PGE2 levels in the hippocampus were also quantified using a PGE2 ELISA kit (highly sensitive kit for inflammation and eicosanoid research, ADI-900-001, Enzo Life Sciences, USA) according to the manufacturer's protocol. Standards were in the range of 2500 to $39.1 \mathrm{pg} / \mathrm{mL}$. Sample PGE2 concentrations were normalized to the wet weight of the piece of tissue analyzed.

\section{Statistical analysis}

Data are reported as means \pm SD. The SPSS software (SPSS 11.5, USA) was used for statistical analysis. Behavioral data were compared by repeated-measures analysis of variance (ANOVA) with post hoc analyses performed after any significant main effect. Two-way ANOVA and post hoc testing were used to compare the Western blot and ELISA data. A value of $\mathrm{P}<0.05$ was considered to be statistically significant.
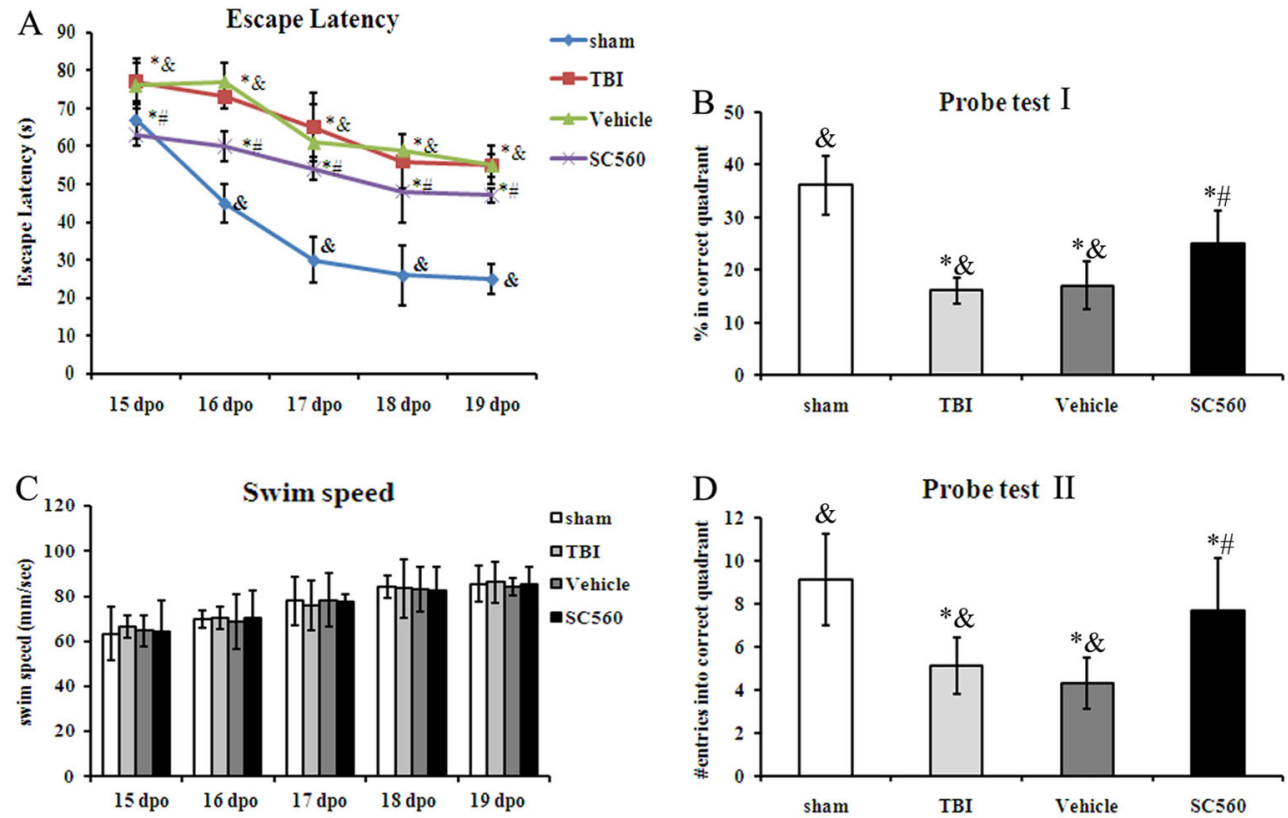

Figure 3. Spatial learning of mice evaluated by the Morris water maze. A, Traumatic brain injury (TBI) mice performed poorly, taking significantly more time to learn the position of the hidden platform compared with the sham group $(\mathrm{P}<0.05)$. After administration with SC560, TBI mice had significant learning improvement on all 5 days of the test (SC560 group vs vehicle group, $\mathrm{P}<0.05$ ). Treatment with SC560 also compensated the defects in both the percent (Probe test I) and the number of entries (Probe test II) into the correct quadrant caused by TBI $(B, D)$. Moreover, there were no differences in swim speed between sham and any of the injured groups $(P>0.05, C)$. The behavior of vehicle-treated TBI mice was similar to that of TBI mice $(P>0.05)$. Data are reported as means $\pm S D$ $(n=8)$. dpo: days post operation. ${ }^{\&} P<0.05$, compared to $S C 560$-treated group; ${ }^{*} P<0.05$, compared to sham group; ${ }^{\#} P<0.05$, compared to vehicle-treated group (repeated-measures ANOVA with post hoc analyses performed after any significant main effect). 

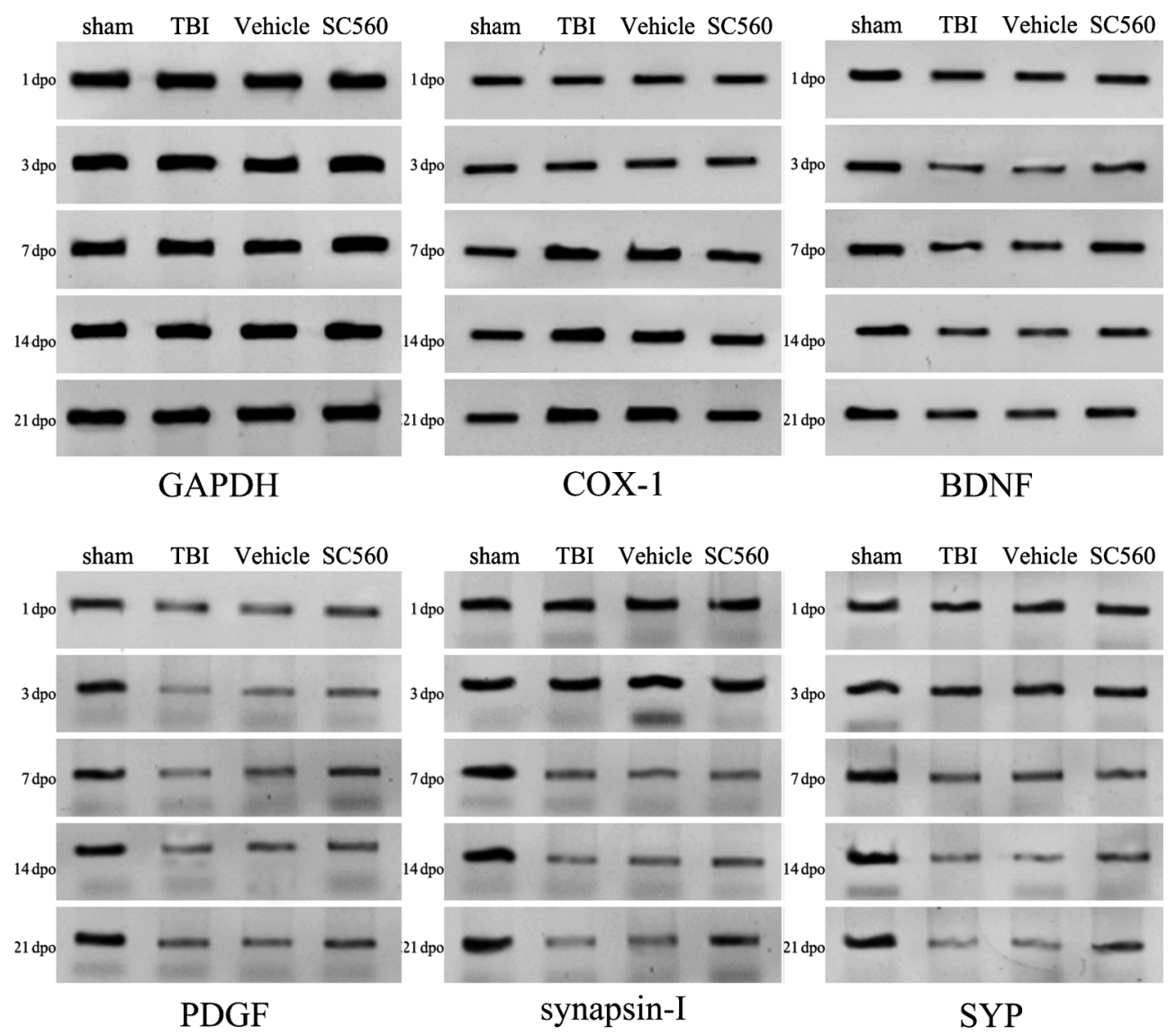

Figure 4. Hippocampal expressions of multiple proteins detected by Western blotting. GAPDH was used as the control. TBI: traumatic brain injury; BDNF: brain-derived neurotrophic factor; PDGF: platelet-derived growth factor BB; SYP: synaptophysin; dpo: days post operation.

\section{Results}

\section{cOX-1 inhibitor treatment improved functional recovery after injury}

Open field. No injury-related changes in open-field behavior were observed among any of the groups (Figure 1).

Beam walk. TBl induced significant deficits in fine motor coordination (sham vs TBI group, $\mathrm{P}<0.001$ ). Analysis by two-way ANOVA revealed that there was a main effect of treatment $\left[P<0.0001, F_{(4,159)}=101.4\right]$, a main effect of time $\left(P<0.0001, F_{(4,159)}=23.43\right)$, and an interaction of treatment and time $\left[P=0.0011, F_{(4,159)}=10.32\right]$. Post hoc tests revealed a significant improvement of foot fault in SC560treated TBI mice compared to the vehicle-treated TBI mice $(P<0.001)$. There was no significant difference between the $\mathrm{TBI}$ group and the vehicle group through the entire experimental period (Figure 2, $\mathrm{P}>0.05$ ).

Morris water maze. Two-way ANOVA analyses revealed that there was a main effect of treatment $(P<0.00001$, $\mathrm{F}=49.52)$, a main effect of time $(\mathrm{P}<0.0001, \mathrm{~F}=32.9)$, and an interaction of treatment and time $(P=0.0011, F=10.32)$. Post hoc tests revealed significant learning impairment in
$\mathrm{TBI}$ mice on all 5 days of the test vs sham $(\mathrm{P}<0.05)$, whereas the behavior of SC560-treated mice was distinguishable from vehicle-treated $T B I$ mice $(P<0.05$, Figure $3 \mathrm{~A}, \mathrm{~B}$, and D). We also observed that SC560treated TBI mice obtained partial restoration in the probe test compared with that of vehicle-treated mice. The SC560 group showed significant increases in both the percentage of time in the correct quadrant (vs vehicle group, $\mathrm{P}<0.05$, Figure $3 \mathrm{~B}$ ) and the number of entries caused by TBI (vs vehicle group, $P<0.001$, Figure $3 \mathrm{D}$ ). All mice had similar swim speeds in the maze (Figure $3 \mathrm{C}$ ). There were no statistical differences between the TBI group and vehicle group $(\mathrm{P}>0.05)$.

\section{Alterations in protein levels induced by SC560 treatment}

Hippocampus was harvested for analysis of expression of multiple proteins, while PB of mice from each group was collected for IL- 6 assays. There was a main effect of treatment $(P<0.0000, F=20.25)$ and a main effect of time $(P<0.0001, F=6.12)$. The post hoc tests revealed the protein levels of $\mathrm{COX}-1$ in the TBI group 

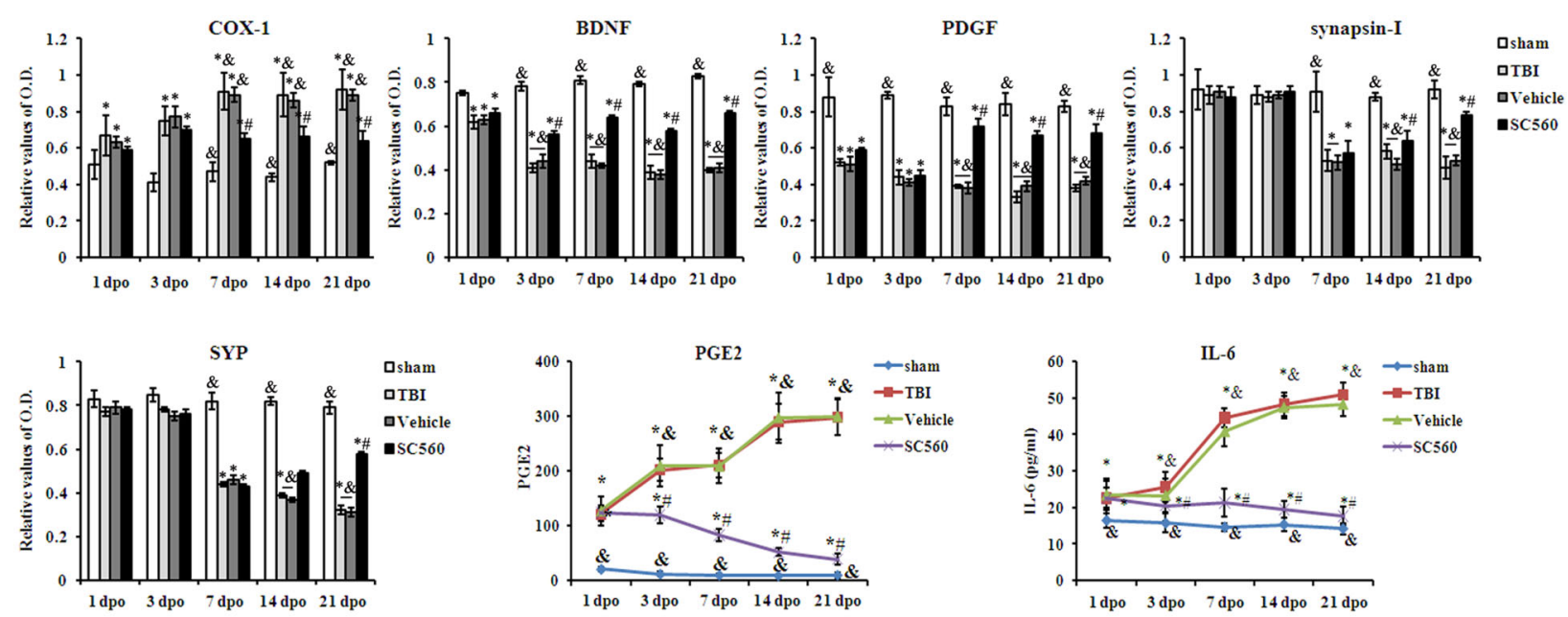

Figure 5. Effect of SC560 treatment on the expressions of multiple proteins. Data showed that compared with the sham group, traumatic brain injury (TBI) induced significant increases in COX-1, prostaglandin E2 (PGE2, pg $\cdot \mathrm{mL}^{-1} \cdot \mathrm{mg}$ tissue $^{-1}$ ) and interleukin (IL)-6 (ELISA), decreases in brain-derived neurotrophic factor (BDNF), platelet-derived growth factor BB (PDGF), synapsin-I and synaptophysin (SYP) protein levels $(P<0.05)$. Systemic administration with SC560 attenuated the increase of COX-1 at 7 dpo as well as that of PGE2 and IL-6 at $3 \mathrm{dpo}$ and reversed the declines of BDNF, PDGF-BB, synapsin-I and SYP protein levels $(P>0.05)$. Data are reported as means $\pm S D(n=8)$. dpo: days post operation; O.D.: absorbance at $280 \mathrm{~nm}$ for Western blot, at $405 \mathrm{~nm}$ for PGE2 ELISA and at $450 \mathrm{~nm}$ for IL-6 ELISA detections, respectively. ${ }^{\text {}} \mathrm{P}<0.05$, compared to SC560-treated group; ${ }^{*} \mathrm{P}<0.05$, compared to sham group; ${ }^{\#} \mathrm{P}<0.05$, compared to vehicle-treated group (two-way AVOVA and post hoc test).

significantly increased at $1 \mathrm{dpo}$, then were raised to the maximal level at $7 \mathrm{dpo}$, and stayed at that highest level to $21 \mathrm{dpo}$, compared with that of the sham-operated group $(P<0.001)$. Similar observations were made for IL-6 expression. There was a main effect of treatment $(P<0.0001, F=9.28)$, a main effect of time $(P<0.0001$, $\mathrm{F}=8.26)$, and an interaction of treatment and time $(P=0.0017, F=3.41)$. Post hoc tests showed the expressions of BDNF and PDGF-BB decreased significantly in the TBI group from 1 to $21 \mathrm{dpo}(\mathrm{P}<0.05)$. Furthermore, synapsin-I and SYP levels decreased beginning at $7 \mathrm{dpo}$ and declined to the lowest level at 21 dpo (Figures 4 and 5, $\mathrm{P}<0.05)$.

Administration of SC560 1) decreased the expression of COX-1 at 7 dpo and of IL-6 and PGE2 at $3 \mathrm{dpo}$; and 2) reversed the decline of $\mathrm{BDNF}$ (at 3, 7, 14, and $21 \mathrm{dpo}$ ) and PDGF-BB (from 1 to $21 \mathrm{dpo}$ ), as well as synapsin-I and SYP (at 7, 14, and $21 \mathrm{dpo}$ ) protein levels (vs vehicle, $\mathrm{P}<0.05$, Figures 4 and 5). However, expression of these proteins did not recover to the levels of the sham-operated group. Additionally, there was no statistical difference in protein expression between the TBI group and the vehicle group $(\mathrm{P}>0.05)$.

\section{Discussion}

The present study showed that the expression of COX1 significantly increased following brain injury in mouse hippocampus. Simultaneously, significant impairment of the beam walk and Morris water maze tasks was observed in the mouse model. TBI also induced an increase in protein expression of COX1, PGE2, and IL-6, as well as a decline in BDNF, PDGF-BB, synapsin-I, and SYP levels. Administration of SC560 following TBI 1) effectively improved performance of beam walk tasks as well as spatial learning and memory; 2) reduced expression of the inflammatory marker IL-6 in PB; and 3) reversed COX-1, PGE2, BDNF, PDGF-BB, synapsin-I, and SYP levels in the hippocampus of injured brains.

Functionally, the present study showed that, in a mouse model, TBI causes deficits in fine motor coordination and spatial learning without gross motor deficits. Moreover, TBI did not affect the swim speed of the mice (8). At the same time, we observed a significant increase in COX-1 and PGE2 hippocampal expression in TBI mice. The present findings suggest that motor coordination as well as learning and working memory are deficient in animals following brain injury and that COX-1, a crucial isoform of the COX family, may be one factor responsible for this phenomenon as described before (10).

The increased accumulation of COX-1 has been identified in different pathologies of the CNS, including ischemic injury, stroke, Alzheimer's disease, and TBI $(5,6,10,11)$. Therefore, the pharmacological inhibition of COX-1 has been shown to be effective in reducing neuronal damage following injury $(5,6,12)$. Recent reports showed that SC560 treatment reduces neuroinflammation neuropathology and improves spatial learning and memory that was impaired due to Alzheimer's disease or lipopolysaccharides $(10,12,13)$. Here, the present data demonstrated 
that treatment with SC560 improved hippocampal-dependent cognitive function, which pointed to the crucial roles of COX-1 in learning and memory loss induced by TBI as described in other research (10).

We also observed an increase in hippocampal expression of PGE2 and IL-6 levels in PB of mice following TBI. IL6 levels have been demonstrated to be effective biomarkers for severity of head injury (14). It has been demonstrated that detrimental effects of inflammatory cells on the brain contributed to the failure of neuroprotective drug trials in TBI (15). It was also reported that COX-1 inhibition by SC560 completely prevented the elevation of PGE2 in irradiated brain (11). We demonstrated that administration of SC560 attenuated the upregulation of COX-1, PGE2, and IL-6 triggered by TBI. It has been shown that the deleterious effects of PGs on working memory are mediated by COX-1 (10). This report also demonstrated that the COX-1-specific inhibitor SC-560 was protective against acute cognitive dysfunction and reduced hippocampal and thalamic PGE2 concentrations (10). Given these factors, we hypothesized

\section{References}

1. Ettenhofer ML, Barry DM. A comparison of long-term postconcussive symptoms between university students with and without a history of mild traumatic brain injury or orthopedic injury. J Int Neuropsychol Soc 2012; 18: 451-460, doi: $10.1017 / \mathrm{S} 1355617711001895$.

2. Ozen LJ, Fernandes MA. Slowing down after a mild traumatic brain injury: a strategy to improve cognitive task performance? Arch Clin Neuropsychol 2012; 27: 85-100, doi: 10.1093/arclin/acr087.

3. Seibert K, Zhang Y, Leahy K, Hauser S, Masferrer J, Perkins W, et al. Pharmacological and biochemical demonstration of the role of cyclooxygenase 2 in inflammation and pain. Proc Natl Acad Sci U S A 1994; 91: 12013-12017, doi: 10.1073/pnas.91.25.12013.

4. Vane JR, Mitchell JA, Appleton I, Tomlinson A, Bishop-Bailey $\mathrm{D}$, Croxtall $\mathrm{J}$, et al. Inducible isoforms of cyclooxygenase and nitric-oxide synthase in inflammation. Proc Natl Acad Sci U S A 1994; 91: 2046-2050, doi: 10.1073/pnas.91.6.2046.

5. Schwab JM, Beschorner R, Nguyen TD, Meyermann R, Schluesener HJ. Differential cellular accumulation of connective tissue growth factor defines a subset of reactive astrocytes, invading fibroblasts, and endothelial cells following central nervous system injury in rats and humans. J Neurotrauma 2001; 18: 377-388, doi: 10.1089/089771501750170930.

6. Schwab JM, Beschorner R, Meyermann R, Gozalan F, Schluesener HJ. Persistent accumulation of cyclooxygenase-1-expressing microglial cells and macrophages and transient upregulation by endothelium in human brain injury. J Neurosurg 2002; 96: 892-899, doi: 10.3171/jns.2002.96.5. 0892.

7. Fox GB, Faden Al. Traumatic brain injury causes delayed motor and cognitive impairment in a mutant mouse strain known to exhibit delayed Wallerian degeneration. J Neurosci Res 1998; 53: 718-727, doi: 10.1002/(SICl)1097-4547 (19980915)53:6<718::AID-JNR9>3.0.CO;2-8. that postinjury inflammatory responses driven by COX-1 were blocked by SC560 treatment, which was responsible for the neuroprotective effects of SC560.

It is known that the NTFs BDNF and PDGF-BB are trophic factors critical for neuronal plasticity, memory, and learning $(16,17)$. Furthermore, BDNF stimulates the synthesis and activation of synapsin-I, resulting in elevated neurotransmitter release and axonal elongation $(10,18)$. Our findings suggested that hippocampal recovery of BDNF and PDGF-BB induced by SC560 treatment attenuated the effects of TBI. SC560 treatment protected the hippocampus from injury-related declines in NTFs and synaptic proteins. Therefore, we postulated that hippocampal COX-1 played a crucial role in cognitive deterioration induced by TBI via inflammation cytokines and NTF regulation, and may be a potential target for drug treatment. However, further investigation is needed.

Taken together, the present study provides new crucial evidence that COX-1 is involved in learning and memory loss induced by TBI in the mouse model.

8. Loane DJ, Pocivavsek A, Moussa CE, Thompson R, Matsuoka Y, Faden Al, et al. Amyloid precursor protein secretases as therapeutic targets for traumatic brain injury. Nat Med 2009; 15: 377-379, doi: 10.1038/nm.1940.

9. Wang TT, Yuan WL, Ke Q, Song XB, Zhou X, Kang Y, et al. Effects of electro-acupuncture on the expression of c-jun and c-fos in spared dorsal root ganglion and associated spinal laminae following removal of adjacent dorsal root ganglia in cats. Neuroscience 2006; 140: 1169-1176, doi: 10.1016/j.neuroscience.2006.03.008.

10. Griffin EW, Skelly DT, Murray CL, Cunningham C. Cyclooxygenase-1-dependent prostaglandins mediate susceptibility to systemic inflammation-induced acute cognitive dysfunction. J Neurosci 2013; 33: 15248-15258, doi: 10.1523/ JNEUROSCI.6361-11.2013.

11. Moore AH, Olschowka JA, Williams JP, Okunieff $P$, O'Banion MK. Regulation of prostaglandin E2 synthesis after brain irradiation. Int J Radiat Oncol Biol Phys 2005; 62: 267-272, doi: 10.1016/j.jijrobp.2005.01.035.

12. Choi SH, Aid S, Caracciolo L, Minami SS, Niikura T, Matsuoka $\mathrm{Y}$, et al. Cyclooxygenase-1 inhibition reduces amyloid pathology and improves memory deficits in a mouse model of Alzheimer's disease. J Neurochem 2013; 124: 59-68, doi: 10.1111/jnc. 12059.

13. Gierse JK, Hauser SD, Creely DP, Koboldt C, Rangwala $\mathrm{SH}$, Isakson PC, et al. Expression and selective inhibition of the constitutive and inducible forms of human cyclooxygenase. Biochem J 1995; 305 (Part 2): 479-484.

14. Hernandez-Ontiveros DG, Tajiri N, Acosta S, Giunta B, Tan $\mathrm{J}$, Borlongan CV. Microglia activation as a biomarker for traumatic brain injury. Front Neurol 2013; 4: 30.

15. Yang SH, Gustafson J, Gangidine M, Stepien D, Schuster $R$, Pritts TA, et al. A murine model of mild traumatic brain injury exhibiting cognitive and motor deficits. J Surg Res 2013; 184: 981-988, doi: 10.1016/j.jss.2013.03.075. 
16. Tropea D, Caleo M, Maffei L. Synergistic effects of brainderived neurotrophic factor and chondroitinase $A B C$ on retinal fiber sprouting after denervation of the superior colliculus in adult rats. J Neurosci 2003; 23: 7034-7044.

17. Ernfors $P$, Bramham $C R$. The coupling of a trkB tyrosine residue to LTP. Trends Neurosci 2003; 26: 171-173, doi:
10.1016/S0166-2236(03)00064-X

18. Valente $P$, Casagrande $S$, Nieus $T$, Verstegen AM, Valtorta F, Benfenati F, et al. Site-specific synapsin I phosphorylation participates in the expression of post-tetanic potentiation and its enhancement by BDNF. J Neurosci 2012; 32: 5868-5879, doi: 10.1523/JNEUROSCI.5275-11.2012. 